\title{
LÚDICA EN AULAS DIFERENCIADAS, UNA PROPUESTA INNOVADORA PARA PROMOVER ACTITUDES FAVORABLES EN EL APRENDIZAJE DE LA QUÍMICA.
}

\author{
Sara Lorena Espejo Villalobos ${ }^{1}$ \\ dqu_sespejo713@pedagogica.edu.co \\ Luisa Fernanda Medina Linares ${ }^{2}$ \\ dqu_lmedina818@pedeagogica.edu.co
}

\begin{abstract}
Resumen
El creciente desinterés mostrado por los estudiantes hacia el aprendizaje de la química, se ha convertido con el tiempo en una de las preocupaciones primordiales de los diferentes actores de la educación; quienes atribuyen la desmotivación a factores como, género, contexto, intereses particulares del estudiante, metodologías de clase, entre otras. En respuesta a estas dificultades, el Gimnasio Fontana (lugar de ejecución); plantea como herramienta de enseñanza, el desarrollo de actividades lúdicas en aulas diferenciadas, para motivar el aprendizaje en los estudiantes desde los diferentes énfasis que el colegio ofrece. Así, en el marco de este trabajo, se aplicaron instrumentos de opinión y observación para detallar los impactos que las estrategias adoptadas por el colegio generan en las dinámicas de clase; encontrándose que la lúdica y la diferenciación reducen en parte la brecha que por la forma, podría generar el trabajo desarrollado de manera independiente en los énfasis.
\end{abstract}

Palabras clave

Diferenciación en el aula, Lúdica, Enseñanza, Aprendizaje, actitudes.

1 Estudiante de noveno semestre, pregrado; Universidad Pedagógica Nacional

2 Estudiante de décimo semestre, pregrado; Universidad Pedagógica Nacional. 


\section{Abstract}

The increasing disinterest showed by the students towards the learning of the chemistry has turned with the time, in one of the primary worries of different actors of the education; those who attribute the desmotivation to factors like kind, context, particular interests of the student, methodologies of class, between others. In response to these difficulties, the fontana Gymnasium (place of execution), it raises as alternative of education, the development of playful activities in differentiated classrooms, in order to motivate the learning in the students from the different emphasises that the college offers. This way, in the frame of this one work, were applied instruments of opinion and observation to detail the impacts that the strategies adopted as the college generate in the dynamics of class; thinking that the playful one and the differentiation reduces partly the gap that by the form, might generate the work developed of an independent way in the emphasis.

Keywords

Differentiation in the classroom, Playful, Teaching, Learning, attitudes

\section{Introducción}

Los resultados obtenidos en la investigación sobre los factores incidentes en las actitudes de los estudiantes en los diferentes énfasis del Gimnasio Fontana (G. F.) Actitudes favorables y desfavorables en el aprendizaje de la química; Una revisión de las implicaciones que los énfasis generan en la disposición de los estudiantes en la clase de química (Espejo, y Medina, 2013); respecto a las intencionalidades inherentes a la metodología por énfasis señala, que las dinámicas de clase desarrolladas en estos espacios es condicionada; en efecto, la enseñanza de la química presenta como variable fundamental la actitud de los estudiantes hacia su aprendizaje. En los estudiantes pertenecientes al énfasis de química y biología y algunos de matemáticas y física, se percibe una mayor disposición hacia el aprendizaje de la química, en relación con los estudiantes de otros énfasis, quienes argumentan que su desmotivación hacia esta asignatura se debe entre otros factores a:

- Descontextualización de los contenidos, con su entorno.

- Aplicabilidad de las teorías en su futuro profesional.

- Las metodologías y estrategias de enseñanza que el profesor emplea en cada una de sus clases.

Este último elemento es el punto de partida para la elaboración de la presente investigación, debido a que, según los resultados obtenidos, las metodologías de clase inciden en las dinámicas de enseñanza-aprendizaje de la química. En consecuencia, se propone el desarrollo e implementación de una estrategia con enfoque lúdico y diferenciado que reconociendo las particularidades de los estudiantes de cada énfasis, potencie actitudes favorables hacia el estudio de esta asignatura.

\section{Descripción del problema.}

¿Son diferenciación y lúdica, estrategias eficaces para potenciar actitudes favorables de los estudiantes en el aprendizaje de la química en un plan educativo regido por énfasis disciplinares?

Teniendo en cuenta la problemática existente en el G. F. respecto a las diferencias actitudinales en el aprendizaje de la química influenciada por los énfasis, se plantea abordar las temáticas de clase por medio de dos herramientas de enseñanza; diferenciación y lúdica en el aula.

Para tal fin, se fijó como objetivo general propiciar, mediante espacios lúdicos y diferenciados, el desarrollo de actitudes favorables hacia el aprendizaje de la química en los estudiantes tanto del énfasis de química y biología como el de artes del Gimnasio Fontana. Para ello se propuso: 
- Diseñar desde un enfoque diferenciado actividades lúdicas como estrategia para generar actitudes favorables hacia el aprendizaje de la química.

- Analizar el comportamiento actitudinal y conceptual de los estudiantes hacia el aprendizaje de la química frente a la metodología propuesta.

\section{Marco teórico}

El G. F. busca potencializar las fortalezas de cada estudiante promoviendo la formación de identidad en cada individuo; (Triana y Zuluaga, 2010) para lo cual establece en su plan de estudios diferentes espacios académicos, en los que se profundiza en determinadas áreas del saber. Tales espacios se denominan énfasis y los estudiantes pueden aplicar a estos, de acuerdo a lo que consideren fundamental para sus posteriores estudios universitarios o simplemente es de su interés (Triana y Zuluaga, 2010).

La educación por énfasis tiene como consigna principal fortalecer la identidad personal y profesional de cada estudiante. Para ello, estructura un plan curricular enfocado en satisfacer las necesidades propias de estos estudiantes dispuestos en grupos, que comparten un mismo objeto de estudio. Ahora, asumir que los estudiantes que cursan un mismo énfasis aprenden de la misma manera y bajo las mismas metodologías es una postura ingenua y en parte cómoda. En consecuencia, el objetivo último del G. F., es proporcionar una educación de calidad; para ello, implementó en sus dinámicas de clase el concepto de diferenciación educativa; entendiéndose diferenciación como el acto consciente de reconocer la pluralidad existente en un grupo de estudiantes (Devalle y Vega, 2006).

Un docente regido por una dinámica diferenciada, se reconoce como un organizador de oportunidades de aprendizaje, cuyo objetivo principal es conocer a cada uno de sus estudiantes. Estos docentes incrementan su capacidad de:
1. Diagnosticar la aptitud del estudiante a través de una variedad de medios.

2. Leer e interpretar los indicios que da el alumno acerca de sus intereses y preferencias en materia de aprendizaje.

3. Crear una diversidad de formas en que los estudiantes puedan explorar e "internalizar" ideas.

4. Presentar distintos canales a través de los cuales los alumnos expresen y amplíen sus conocimientos (Tomlinson, 2005).

Entre la diversidad de estrategias disponibles se retoma la lúdica como estrategia favorable para la enseñanza de la química.

La lúdica como una estrategia comunicativa, cognitiva, afectiva, y/o de memorización que está directamente relacionada con el aumento de las actitudes positivas de los estudiantes en el aprendizaje logrando cautivar su interés al crear sus propias estrategias obteniendo un mayor razonamiento y comprensión de las temáticas al orientar la producción de conocimiento, además de fomentar el buen desarrollo psicosocial (Ballesteros, 2011 Hernán y Gabriel, 1998, Bolívar, 1998, Martínez, 2008).

Por otra parte, debido a la multiplicidad de interpretaciones que la literatura ofrece acerca del término actitud, en este documento se retoma a Shaw y Wright (citados en Escudero, 1985) quienes definieron la actitud como "un sistema relativamente duradero de reacciones afectivo-evaluativas, basadas en conceptos evolutivos o creencias aprendidos acerca de las características de un objeto social". 


\section{Metodología}

Este proyecto se efectúo en dos partes o etapas fundamentales que se describen a continuación (Esquema 1).

Esquema 1. Etapas de la investigación.

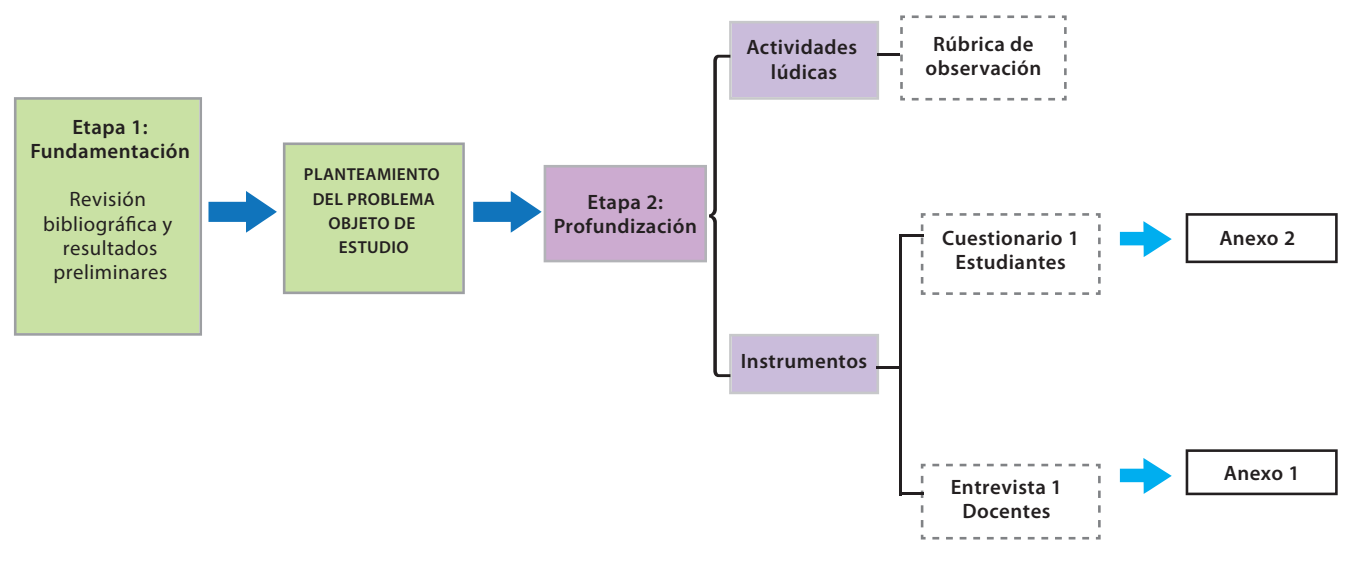

\section{Resultados}

\section{Población objeto de trabajo}

Siguiendo las pautas descritas en la metodología, se plantean los resultados obtenidos en cada instrumento. En esta medida el trabajo se divide en dos etapas; en la primera se enuncia los resultados obtenidos en el cuestionario aplicado a los estudiantes de décimo (gráficas 1, 2, 3 y 4 ) y undécimo grado (gráficas 5, 6, 7 y 8 ) mediante gráficos de barras; en un segundo momento se plantea el destilado de las entrevistas realizadas a los docentes del área de química del G. F (Tabla 2).

Tabla 1. Población participante, Grado décimo y undécimo.

\begin{tabular}{|c|c|c|}
\hline Curso & Node personas & Porcentaje \\
\hline $10-A$ & 18 & $18.2 \%$ \\
\hline $10-B$ & 17 & $17.2 \%$ \\
\hline $10-C$ & 11 & $11.1 \%$ \\
\hline $10-D$ & 13 & $13.1 \%$ \\
\hline $11-A$ & 14 & $14.1 \%$ \\
\hline $11-B$ & 6 & $6.1 \%$ \\
\hline $11-C$ & 11 & $11.1 \%$ \\
\hline $11-D$ & 9 & $9.1 \%$ \\
\hline
\end{tabular}

Resultados encuesta, Grado décimo. Gráfica 1. Primera Pregunta.

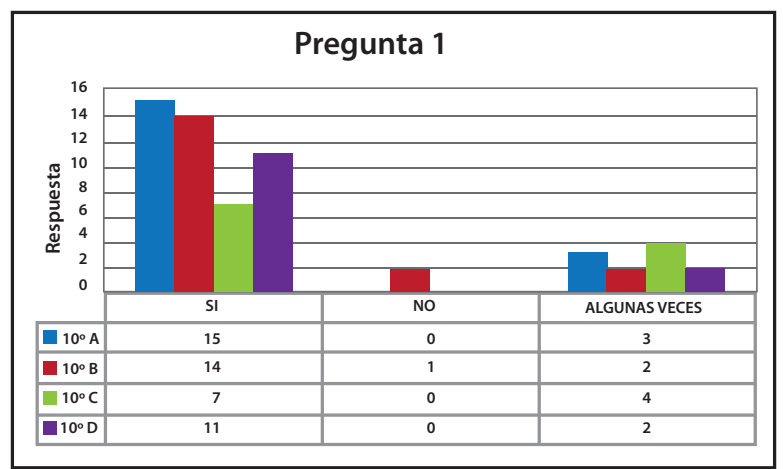

Gráfica 2. Segunda Pregunta.

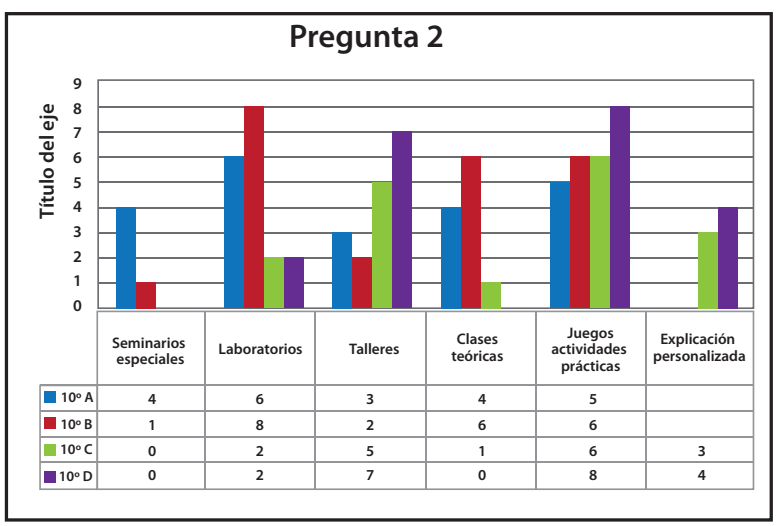


Gráfica 3. Tercera Pregunta.

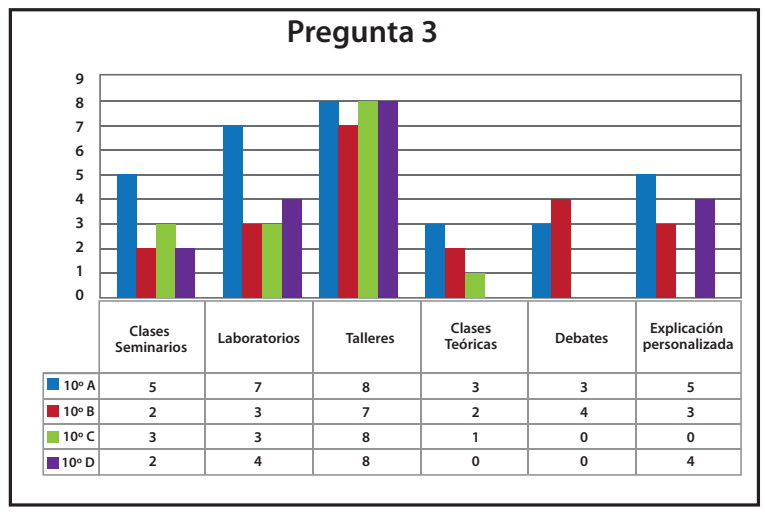

Gráfica 4. Cuarta Pregunta.
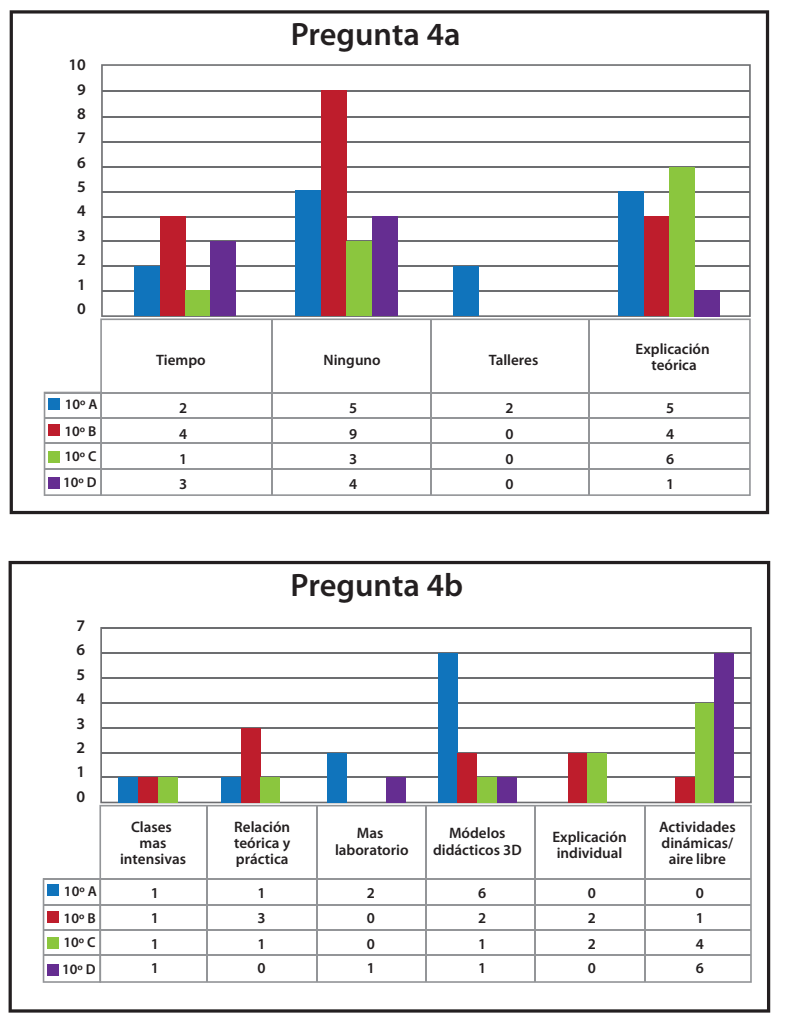

Resultados Encuesta, Grádo undécimo. Gráfica 5. Primera Pregunta.

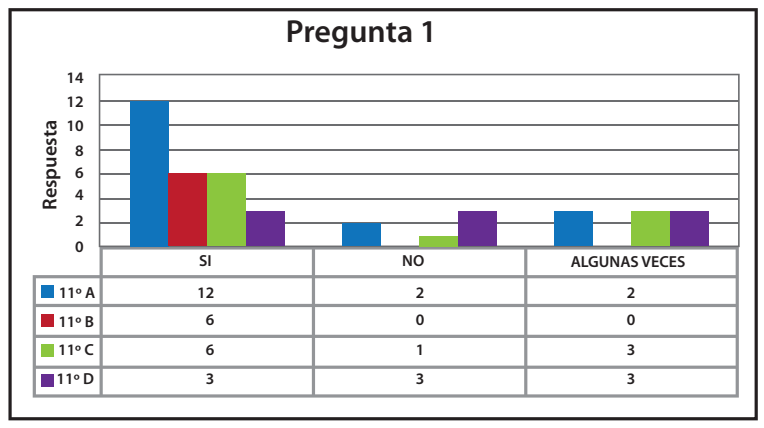

Gráfica 6. Segunda Pregunta.

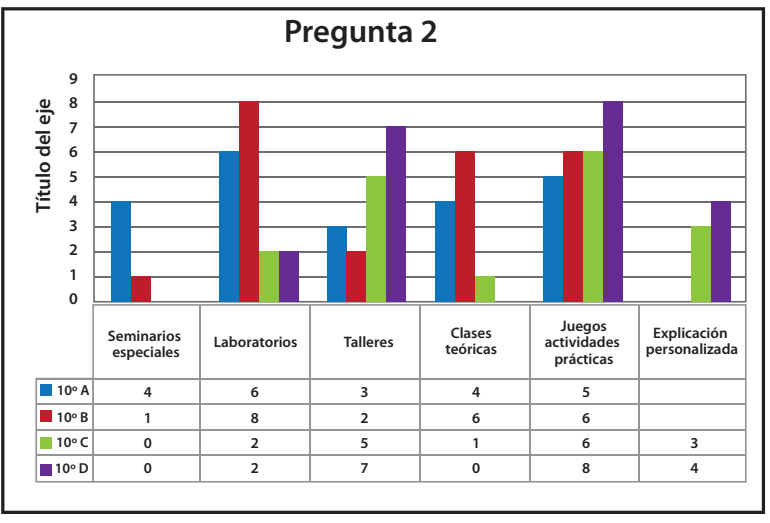

Gráfica 7. Tercera Pregunta.

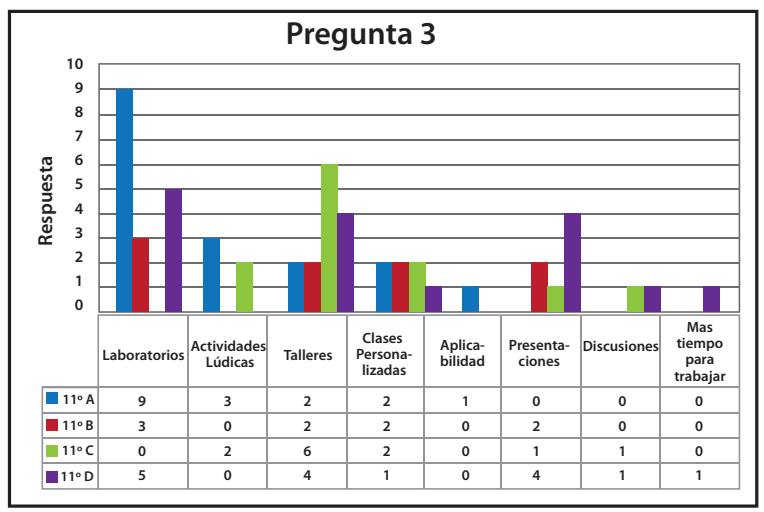


Gráfica 8. Cuarta Pregunta

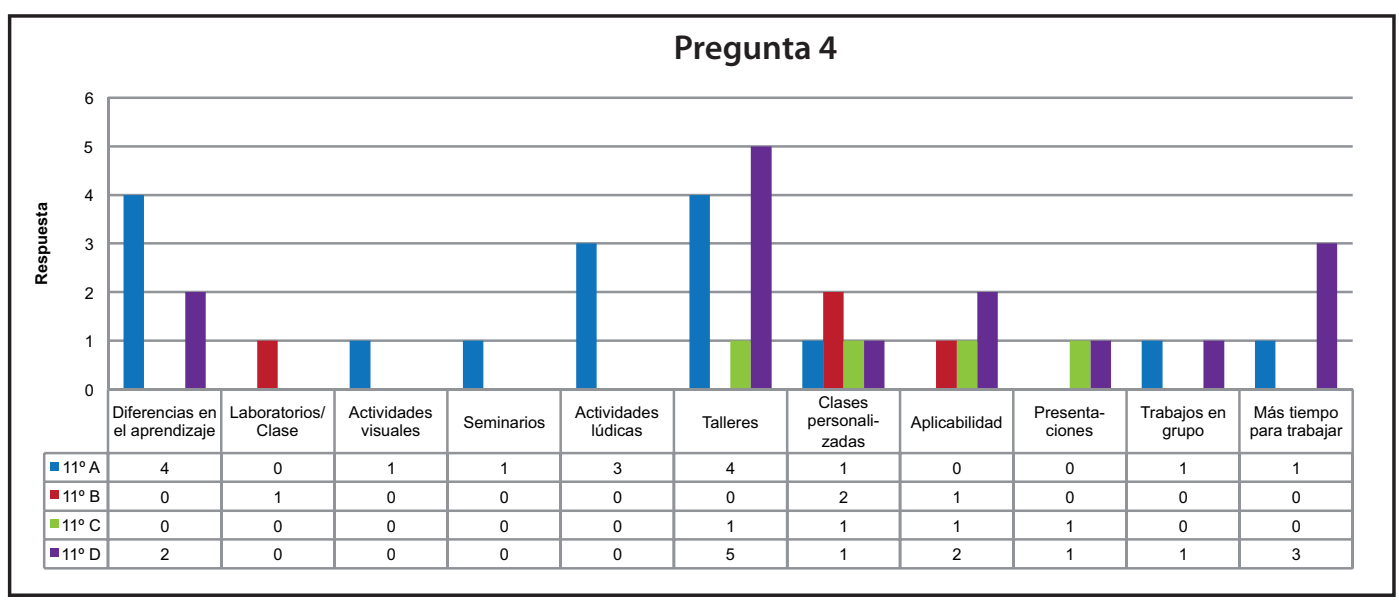

Tabla 2. Entrevista Docente G. F.

\begin{tabular}{|c|c|c|}
\hline $\begin{array}{l}\text { UNIDADES DE } \\
\text { INFORMACIÓN }\end{array}$ & Prof. John Luna & Prof. Pilar Castillo \\
\hline PAEC1-1 & $\begin{array}{l}\text { Es la manera de hacer bien la } \\
\text { pedagogía }\end{array}$ & $\begin{array}{l}\text { Uso de estrategias didácticas para potenciar } \\
\text { el desarrollo del estudiante, la lúdica incluye } \\
\text { el juego }\end{array}$ \\
\hline PAEC1-2 & $\begin{array}{l}\text { Cuando haces motivación e } \\
\text { innovación }\end{array}$ & $\begin{array}{l}\text { Cuando se planean actividades que incluyan } \\
\text { diferentes tipos de inteligencias }\end{array}$ \\
\hline PAEC1-3 & $\begin{array}{l}\text { Si porque permite apropiar los } \\
\text { conceptos y actividades }\end{array}$ & $\begin{array}{l}\text { Si por que entre mayor cantidad de } \\
\text { actividades que tenga el docente, entenderá } \\
\text { los diferentes estilos de aprendizaje }\end{array}$ \\
\hline PAEC1-4 & $\begin{array}{l}\text { Positivo, ya que identifican } \\
\text { diferencias en el aprendizaje. Si, ya } \\
\text { que el hecho de que los estudiantes } \\
\text { no escojan el énfasis por ciertos } \\
\text { intereses implica que muestres } \\
\text { barreras que impiden su aprendizaje }\end{array}$ & $\begin{array}{l}\text { Normalmente los estudiantes del énfasis } \\
\text { están mas dispuestos y con mejor actitud } \\
\text { que los de la básica }\end{array}$ \\
\hline PAEC1-5 & $\begin{array}{l}\text { No, tal vez en el tiempo que se } \\
\text { requiere para desarrollar las } \\
\text { actividades }\end{array}$ & No \\
\hline PAEC1-6 & $\begin{array}{l}\text { Identificando dificultades y fortalezas } \\
\text { en cada una de las actividades } \\
\text { propuestas }\end{array}$ & $\begin{array}{l}\text { Implementando trabajo colaborativo, } \\
\text { diferentes estrategias, meta cognición y } \\
\text { evaluación entre pares }\end{array}$ \\
\hline PAEC1-7 & $\begin{array}{l}\text { Es útil para identificar y apropiar los } \\
\text { diferentes niveles de aprendizaje }\end{array}$ & $\begin{array}{l}\text { No solo en química, si no en todas las áreas } \\
\text { ya que "personaliza" y potencializa el } \\
\text { desarrollo y el proceso de aprendizaje del } \\
\text { estudiante. }\end{array}$ \\
\hline PAEC1-8 & $\begin{array}{l}\text { Pienso que los compromisos se } \\
\text { adquieren a medida que avanza el } \\
\text { aprendizaje }\end{array}$ & $\begin{array}{l}\text { Conocer estrategias de diferenciación y } \\
\text { aplicar de acuerdo al diagnostico del grupo }\end{array}$ \\
\hline PAEC1-9 & $\begin{array}{l}\text { Influye en el camino que tienes frente } \\
\text { a la manera de aprender }\end{array}$ & $\begin{array}{l}\text { Influye en el camino que tienes frente a la } \\
\text { manera de aprender }\end{array}$ \\
\hline
\end{tabular}




\section{Análisis de resultados.}

Antes de examinar los resultados obtenidos tras la aplicación del cuestionario en cada grupo, es fundamental resaltar la población, específicamente el contexto en el que se aplicó el instrumento. Esto debido a que, antes de la actividad, se efectuaron las clases y actividades planeadas y no planeadas desde el enfoque de este proyecto. Hecho que incide de cierta forma en la percepción de los estudiantes y por ende en la respuesta de los mismos. Adicional a ello, es importante señalar que las clases realizadas están diseñadas desde la metodología por énfasis que se maneja en la institución, lo cual genera otras consideraciones desde el punto de vista disciplinar y actitudinal.

Hecha esta salvedad y retomando los resultados obtenidos en la aplicación del primer instrumento dirigido a los estudiantes de décimo y undécimo grado del G. F., específicamente en la primera pregunta (gráfica 1 y 5); se infiere, en términos generales, que las guías, actividades y metodologías propuestas por parte de los docentes titulares y docentes practicantes responden a los diferentes tipos de aprendizaje de los estudiantes. Aun así, señala un porcentaje minoritario de estudiantes; se requiere un mayor esfuerzo por parte de los docentes a la hora de reconocer y actuar en función de los diferentes tipos de aprendizaje de los estudiantes pertenecientes a un mismo énfasis, debido a que no todos aprenden del mismo modo y en el mismo periodo de tiempo, lo que incide en el proceso la motivación y disposición hacia la clase.

Ahora bien, de los aportes realizados por los estudiantes encuestados se puede analizar dos cuestiones fundamentales en la construcción del presente proyecto; en primera instancia, se destaca la importancia de la lúdica en la motivación hacia la clase de química. De hecho, los estudiantes manifiestan que "el desarrollo de clases más lúdicas y dinámicas, contribuyen a aumentar el interés por la clase, facilitando la relación de la teoría vista con la practicidad de la misma". También se hace evidente la dificultad inmanente a la comprensión de las temáticas abordadas en cada grado; por ejemplo, el énfasis en química y biología, tanto de 10 como de 11 retoma contenidos más elaborados y complejos, que demandan un esfuerzo adicional en la elaboración de talleres y actividades en relación con los otros énfasis, de los cuales el énfasis de Filosofía y sociales (10 C y $11 \mathrm{C}$ ) presentó mayor dificultad. Ante esta problemática, se optó por incrementar las explicaciones individuales o clases personalizadas en este grupo, aprovechando el reducido número de estudiantes, lo que arrojó resultados satisfactorios reflejados en la fluidez de los mismos al explicar ejercicios y en sus calificaciones.

Esta dificultad, seguida de la pluralidad de intereses existente entre los estudiantes de un mismo énfasis y la necesidad de motivar a estos sujetos hacia el aprendizaje de la química, facilitó el desarrollo de algunas estrategias que en la medida de las posibilidades permitió atesorar la motivación de los estudiantes del énfasis de química y biología hacia el aprendizaje de la química e incentivar a los integrantes de los énfasis restantes hacia este proceso. Los estudiantes del énfasis de química y biología (10 A y $11 \mathrm{~A}$ ), debido a las intencionalidades de la institución como se describió anteriormente en este documento y dada la naturaleza de las actividades propuestas, tuvieron mayor participación en su ejecución en relación con los otros énfasis; asimismo, las actividades estuvieron diferenciadas por contenido, proceso y producto. $\mathrm{A}$ continuación se describe algunas de las actividades desarrolladas, que según los estudiantes permiten o facilitan el aprendizaje de la química.

Las temáticas abordadas en el colegio se enmarcan en tres núcleos integradores correspondientes a los tres periodos académicos, el primero de ellos en el cual se trabajó a profundidad, es el cuerpo humano; todas las actividades están relacionadas con este núcleo, buscando con ello mostrarle a los estudiantes, la importancia de las diferentes asignaturas en su cotidianidad. En el caso de química, se procuró mostrar la importancia de la química en los alimentos que ingerimos, en las reacciones bioquímicas y en contexto; empleando, talleres contextualizados, prácticas de laboratorio, seminarios especiales, exposiciones orales y actividades lúdicas. 
En los talleres se integra el componente procedimental respecto a la elaboración de ejercicios de tipo lápiz y papel con situaciones problema desde diferentes tipos de pregunta, en donde se diferencia por contenidos la temática abordada, buscando con ello proporcionar al estudiante diferentes rutas para comprender el concepto trabajado. Por ejemplo, para el tema de estequiometría se realizó una aproximación empleando tuercas y tornillos; de esta actividad se resalta que los estudiantes muestran mayor interés en el aprendizaje de la química al realizar procedimientos diferentes a los establecidos en el libro de texto; asimismo, que este tipo de actividades exige diferentes habilidades lo cual permite una integración de los estudiantes con el fin de realizar la tarea solicitada. Otras actividades realizadas bajo este enfoque, fueron la preparación de postres empleando el concepto de reactivo límite y cambios químicos de la materia, preparación de soluciones y unidades físicas de concentración a través de la preparación de bebidas a base de frutas en polvo, análisis de exámenes médicos y las implicaciones a la salud de los resultados que facilitaron la comprensión de la temática abordada.

Los trabajos prácticos de laboratorio también desempeñaron un papel fundamental en el objetivo de este proyecto, por una lado (grado 11), permitieron recrear parte de la teoría retomada en su momento mediante reacciones químicas en el laboratorio $y$, por otro (grado 10), permitieron que los estudiantes se aproximaran a algunas aplicaciones industriales de la química en la elaboración de productos de uso diario como gel reductor térmico, champú, gel antibacterial, betún, obtención de esencias, entre otros. Esta última actividad requirió analizar como los reactivos que intervienen en dicha elaboración tienen un impacto ambiental y sintomatológico en el cuerpo humano, por lo que pueden adquirir una visión de la química aplicada, que tiene relación con su entorno y experiencias diarias incidiendo en la curiosidad por descubrir el funcionamiento de muchos más reactivos químicos compuestos orgánicos con grupos funcionales específicos como esteres, alcoholes, ácidos carboxílicos y de la química en general potenciando su interés por el aprendizaje de la química.
Otro componente fundamental, son los seminarios especiales; donde a través de temas como la química del chocolate, armas químicas, la química en tu perfume, la química del amor, entre otros, los estudiantes evidencian por medio de sus sentidos propiedades como olor, sabor y textura de reactivos químicos con los que ellos tienen contacto a diario, por lo cual adquieren un lenguaje diferente y empiezan a ver relación de la química en toda su vida. Lo anterior, propicia el desarrollo de su curiosidad e interés por el aprendizaje de la química, cumpliendo así uno de los objetivos de la diferenciación, donde el docente organiza y abre espacios en los cuales el estudiante tiene, de formas diferentes, variadas oportunidades de adquirir conocimiento.

Por otra parte las exposiciones orales, como la explicación de la cinética presente en los motores y la degradación de la capa de ozono, posibilitan que los estudiantes se apropien de una temática y a través de su creatividad compartan con sus compañeros su postura frente a un tema específico. De esta actividad, se destaca que los estudiantes de la básica (de los énfasis), realizan actividades, de forma y fondo más elaboradas. Finalmente, las clases realizadas, tanto por los profesores titulares como en formación inicial, buscaban explicar la temática sin caer en las dinámicas de transmisión-recepción, razón por la cual, las actividades de este tipo solo demandaban un $20 \%$ de la hora de clase.

Teniendo en cuenta las intencionalidades de este proyecto, se analizan dos posturas diferenciadas en las dinámicas de clase en grado décimo y undécimo (Tabla 2). En primera instancia, el profesor de química del grado decimo presenta una concepción limitada respecto a la lúdica; en cuanto a definición, utilidad y ejecución, impidiendo que se aprovechen dichas herramientas eficazmente. Esta postura se refleja en la afirmación emitida por el mismo, "los tiempos no son suficientes para abordar las temáticas propias de la clase, de manera conjunta con la lúdica y diferenciación", entonces el profesor entiende la lúdica como actividades dinámicas que no pueden estar implícitas en el desarrollo y tiempos de la clase de química. Ahora bien, es importante resaltar que las clases planeadas desde la articulación de las temáticas con la lúdica y la dife- 
renciación, tuvieron un desarrollo exitoso; demostrando finalmente que los tiempos destinados a el cumplimiento durante el desarrollo de las clases no se ven afectados ni deben ser un limitante para la implementación de dichas estrategias. Por lo que es esencial para cualquier docente tener claras las herramientas que se emplean en el aula, lo que beneficiará en mayor medida a los actores principales de la clase.

Por otra parte, al abordar el tema de la diferenciación en el aula, el docente afirma que "es útil para identificar y apropiar los diferentes niveles de aprendizaje", sin embargo no se habla de la intervención en las diferencias encontradas en el aprendizaje de cada estudiante; en este orden de ideas, no hay claridad en cuanto a los compromisos que adquiere el docente al implementar la diferenciación en el aula.

En segunda instancia en la entrevista realizada a la profesora de química de grado once, se resaltan dos puntos; primero se tiene una idea clara del concepto de lúdica y las implicaciones que las metodologías planeadas desde este enfoque demandan por parte del docente, y genera en los estudiantes. Segundo, la docente está familiarizada con la herramienta didáctica de diferenciación y con la importancia de reconocer las diferencias propias de un grupo en el proceso de enseñanza-aprendizaje, ya que "personaliza" y potencializa el desarrollo y el proceso de aprendizaje del estudiante". Para lo cual, señala, es fundamental realizar "un diagnóstico previo, desarrollar actividades variadas, trabajo colaborativo y observación detallada de los procesos".

En su mayoría, las actividades lúdicas como se describe en la tabla 4 y 8 , se enfocan en el énfasis de química y biología; en consecuencia, las opciones de los énfasis restantes están limitadas por la clasificación que el trabajo por "énfasis" genera. Condición que restringe las dinámicas de trabajo haciéndolas monótonas y enfocadas a la mera adquisición de conocimientos. Todo lo anterior justifica que algunos estudiantes se muestren inconformes, y soliciten actividades lúdicas, laboratorios y mayor acompañamiento.
Esto último, da peso a los esfuerzos que el colegio y los docentes realizan a diario por diferenciar a sus estudiantes y responder a sus necesidades, de tal forma que estos puedan apropiar los diferentes conceptos trabajados en clase de la mejor manera posible.

\section{Conclusiones.}

Diferenciar, desde una perspectiva educativa, se puede entender como el reconocimiento de una pluralidad inherente a la conducta humana que, al permear el proceso educativo potencia y favorece el aprendizaje significativo de los contenidos.

En este orden de ideas, la lúdica entendida como estrategia comunicativa, cognitiva, afectiva, y/o de memorización, está directamente relacionada con el aumento de las actitudes positivas de los estudiantes de los diferentes énfasis hacia el aprendizaje; y estas, al ser planeadas diferenciadamente (con objetos distintos) en la enseñanza de la química, permiten estimular algunas habilidades específicas adaptadas a las necesidades del espacio académico con que se cuenta para enseñar química en una institución.

No obstante, esta estrategia educativa está supeditada a las necesidades del colegio; puntualmente el trabajo por énfasis, los intereses particulares de los estudiantes y los fundamentos disciplinares, pedagógicos y didácticos que respaldan la práctica docente y a su vez se relaciona con el criterio que emplean los profesores para asumir el juego y los recursos didácticos empleados para su realización, enfocados a contribuir a la construcción del conocimiento con los niños que asisten a la escuela (Duarte, 2003). Desde esta perspectiva Erick de Corte presenta, en su investigación los diferentes aportes que las ciencias de la mente han realizado al mejoramiento de la práctica educativa, por medio de tres preguntas:

¿Qué tipos de conocimientos, estrategias cognitivas y cualidades afectivas deben ser aprendidos, de manera que los alumnos tengan disposición para aprender a pensar y resolver problemas con 
habilidad? ¿Qué tipo de procesos de aprendizaje deben ser llevados a cabo por los alumnos para lograr la pretendida disposición, incluyendo la mejora de categorías de conocimientos y habilidades? Y, ¿cómo pueden crearse ambientes de aprendizaje lo suficientemente dinámicos y poderosos para lograr en los alumnos una disposición para aprender a pensar activamente? (Duarte, 2003, p.100)

Investigaciones desarrolladas sobre el juego, (Ferrari 1994) destacan la gran variedad de propósitos dentro del contexto de aprendizaje. Señalan además que dos de sus potencialidades básicas, son la posibilidad de construir autoconfianza y la de incrementar la motivación en el jugador. Es un método eficaz que posibilita una práctica significativa de aquello que se aprende; el juego en la educación ha servido como motivador y a veces como recurso didáctico, aspectos encontrados en esta investigación.

\section{Referencias bibliográficas.}

Ballesteros, O. (2011). La lúdica como estrategia didáctica para el desarrollo de competencias científicas. (Tesis de maestría).Universidad nacional de Colombia, Bogotá D.C. Recuperado de http:// portales.puj.edu.co/ftpcentroescritura/Recursos/Normasapa.pdf

Bolívar. C (1998). Aproximación a los conceptos de lúdica y ludopatía. Ponencia presentada en el V Congreso Nacional de Recreación Coldeportes Caldas / Universidad de Caldas / FUNLIBRE, Manizales, Colombia. Recuperado de http://www.redcreacion. org/documentos/congreso5/CBolivar.htm

Devalle, A. y Vega, V. (2006). Una escuela en y para la diversidad. El entramado de la diversidad. Buenos Aires: Paidós Ibérica.

Duarte, J. (2003). Ambientes de aprendizaje: Una aproximación conceptual. Estudios pedagógicos, (29), $97-113$.

Escudero, T. (1985). Las actitudes en la enseñanza de las ciencias: un panorama complejo. Revista de Educación, 2(78), 5-25.
Espejo, S. y Medina, L. (2013). Actitudes favorables y desfavorables en el aprendizaje de la química: Una revisión de las implicaciones que los énfasis curriculares generan en la disposición de los estudiantes hacia la clase de química. Artículo elaborado en el marco del proyecto de práctica docente I en el Gimnasio Fontana. (Documento sin publicar) Bogotá: Colombia.

Ferrari, C. (1994). ¿Qué es el juego? Alrededor del fútbol. Revista Universidad de Antioquia, 63(236), 47-49.

Hernán, J. y Gabriel, J. (1998). Lo lúdico como componente de lo pedagógico, la cultura, el juego y la dimensión humana. Ponencia presentada en: $V$ Congreso Nacional de Recreación Coldeportes Caldas / Universidad de Caldas / FUNLIBRE, Manizales, Colombia. Recuperado de http://blog.utp.edu.co/ areaderecreacionpcdyr/files/2012/07/LO-LUDICO-COMO-COMPONENTE-DE-LO-PEDAGOGICO.pdf

Martínez, L. (2008). Lúdica como estrategia didáctica. Recuperado de http://genesis.uag.mx/escholarum/vol11/ludica.html

Tomlinson, C. (2005). Estrategias para trabajar con diversidad en el aula. Buenos Aires: Paidós Ibérica.

Triana, A. y Zuluaga, C. (2010). Educar la condición humana. ¿Una revolución educativa? Bogotá. Colombia. 


\section{Anexos.}

\section{Instrumento 1.}

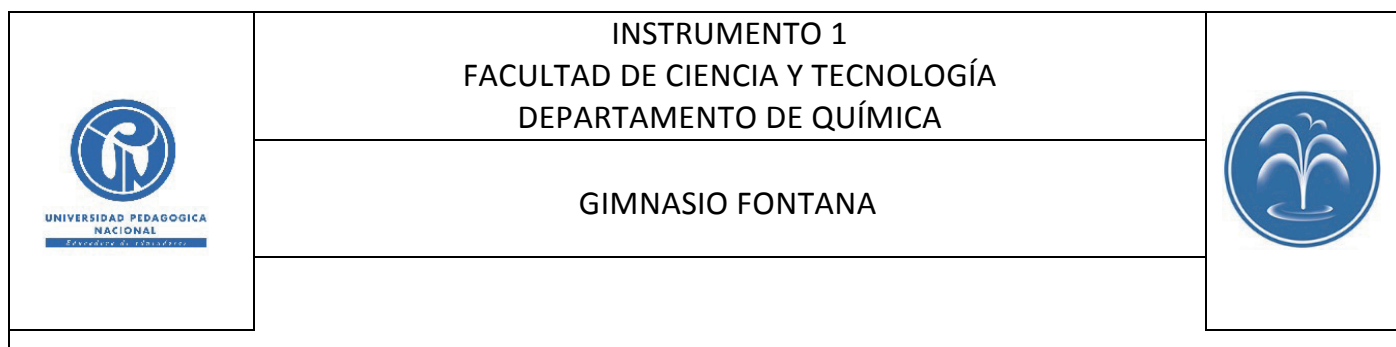

Esta entrevista está constituida por nueve preguntas abiertas, que pretenden identificar y analizar la percepción de los docentes de química del GF respecto a la lúdica como estrategia de enseñanza en el aula. Así mismo, indagar por la concepción de aprendizaje particularmente en las medidas que se retoman para propiciar actitudes favorables hacia el aprendizaje de la química.

Entrevista elaborada en el marco del proyecto de práctica pedagógica II; Lúdica y diferenciación como estrategia didáctica para fomentar actitudes favorables en el aprendizaje de la química.

1. ¿Qué entiende por boca?

2. ¿Cuando se hace lúdica en el aula?

3. ¿Considera usted que la lúdica puede ser una estrategia útil para la enseñanza de la química? ¿Por qué?

4. Cuando se explica un tópico en química a través de una actividad lúdica, ¿Cuál es el comportamiento de los estudiantes en los diferentes énfasis? Es decir, ¿̇se puede hablar de una tendencia en el comportamiento de los estudiantes frente a la actividad?

5. ¿Ha evidenciado alguna desventaja en la implementación de la lúdica? ¿Cual?

6. ¿Cómo aproximar a tos estudiantes que hacen parte de un mismo curso al aprendizaje de la química en el aula?

7. ¿La diferenciación es una herramienta útil para el proceso de la enseñanzaaprendizaje de la química? ¿En qué forma?

8. ¿Que compromisos adquiere como profesor de química al implementar diferenciación en el aula?

9. Cuando se realiza diferenciación en los estudiantes ¿Cómo influye el componente actitudinal de los estudiantes en dicha diferenciación? 


\section{Instrumento 2.}

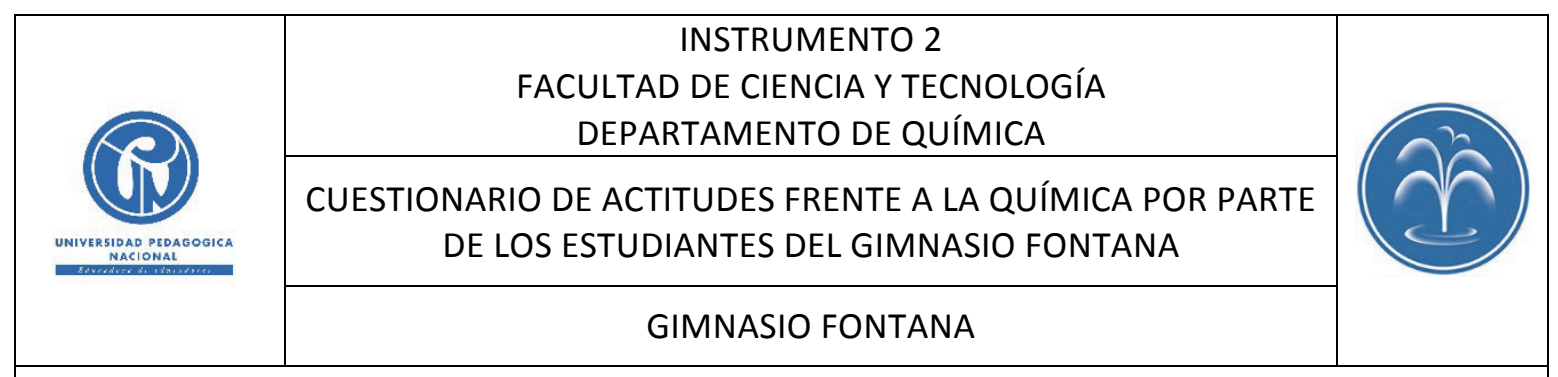

Esta entrevista está constituida por cuatro preguntas abiertas, que pretenden identificar y analizar la percepción de los estudiantes de los grados décimo y undécimo del GF respecto a la lúdica como estrategia de enseñanza en el aula. Así mismo, indagar por la concepción de aprendizaje particularmente en las medidas que se retoman para propiciar actitudes favorables hacia el aprendizaje de la química.

Entrevista elaborada en el marco del proyecto de práctica pedagógica II; Lúdica y diferenciación como estrategia didáctica para fomentar actitudes favorables en el aprendizaje de la química.

1. ¿En la clase de química evidencia usted que los temas, las guías y la forma de explicación, están proyectados teniendo en cuenta los diferentes tipos de aprendizaje de los estudiantes?

2. ¿Qué actividades cree usted que le permite adquirir mayor conocimiento en una clase de química?

3. ¿Dé cuenta de alguna metodología (actividades de clase) que le haya permitido aplicar, analizar, argumentar y comprender una temática trabajada en clase de química?

4. ¿Qué dificultades ha tenido usted en el aprendizaje de alguna temática química mediante una clase tipo magistral?, ¿qué propondría para el mejoramiento de la clase? 